\title{
Is Electrocochleography Still Helpful in Early Diagnosis of Meniere Disease?
}

\author{
Fulvio Mammarella ${ }^{1}$, Melissa Zelli ${ }^{1}$, Theodoros Varakliotis ${ }^{1,2}$, Alberto Eibenstein ${ }^{2}$, \\ Claudio Maria Pianura ${ }^{1}$, and Gianluca Bellocchi ${ }^{1}$ \\ ${ }^{1}$ ENT Department, San Camillo Forlanini Hospital, Rome, \\ ${ }^{2}$ Department of Applied Clinical Sciences and Biotechnology (DISCAB), L'Aquila University, L'Aquila, Italy
}

Received December 9, 2016

Revised March 5, 2017

Accepted March 30, 2017

Address for correspondence

Theodoros Varakliotis, MD

Department of Applied Clinical

Sciences and Biotechnology

(DISCAB),

L'Aquila University,

Via Vetoio (Coppito 2),

Coppito, L'Aquila 67100, Italy

Tel +39-065506749

E-mail theo_va@hotmail.com
Background and Objectives: Despite the extensive research for the Meniere's disease (MD), it's pathophysiology still remains uncertain and questionable among scientists. Clinical symptoms and audiometric tests form the basis for the diagnosis. Nevertheless the differential diagnosis can be extremely challenging, due to subjective and not specific results. Incorrect diagnosis is most likely and for this reason there is a great demand for objective and reliable tests. Subjects and Methods: The presence of endolymphatic hydrops is necessary condition but non enough for the diagnosis. In this study we analyze retrospectively the summating potentials (SP)/action protentials (AP) ratio from peritympanic electrocochleography in 34 patients, divided in two groups (13 patients classified as defined MD and 21 patients classified as probable MD). The purpose was to identify the utility of the exam so to be able to achieve an early defined diagnosis. Furthermore our intention was to obtain an objective test, besides the clinical criteria currently in use for the diagnosis and the classification of the MD. Results: The analysis of the SP/AP results shows high predictability for the MD (positive in six out of seven cases with a sensitivity greater than $80 \%)$. Conclusions: Our study demonstrates a satisfactory sensibility percentage in recurrent defined MD presentation ( $>80 \%$ ). Early identification of MD can lead to an early treatment and control of the progression of the disease. Furthermore it could be a valuable instrument for the follow up and evaluation of the patients classified as probable MD in accordance with the criteria of the American Academy of Otolaryngology-Head and Neck Surgery (1995). J Audiol Otol 2017;21(2):72-76

KEY WORDS: Meniere's disease · Endolymphatic hydrops · Electrocochleography.

\section{Introduction}

The incidence of Meniere's disease (MD) in the general population is slightly above $0.2-0.4 \%$ with complete presence of the three characteristic symptoms (tinnitus, hearing loss and vertigo) in only $40 \%$ [1] and an high prevalence of incomplete manifestations that make the disease harder to diagnose in early stages. The presence of cochleopathy, often characterized by the association of sensorineural hearing loss and tinnitus, could raise the suspicion of endolymphatic hydrops, especially when there is evidence of fluctuations in the hearing

This is an Open Access article distributed under the terms of the Creative Commons Attribution Non-Commercial License (http://creativecommons.org/licenses/by-nc/4.0/) which permits unrestricted non-commercial use, distribution, and reproduction in any medium, provided the original work is properly cited. threshold [1]. Recent studies, however, tend to associate patients with low frequency tone hearing loss and absence of dizziness with a reduced progression in defined MD $(<9 \%)$ [2]. This is confirmed in the literature (12.2\%) [3] by the predominance of flat audiometric curves in patients with definite diagnosis of MD [4]. On the contrary, it is still underestimated the initial vestibular symptomatology which can be absent at the instrumental investigations during the initial stages [5]. It is indeed the first primary cause of medical and surgical treatment at later stages. The reasons are multiple: the finding of normal reflectivity in patient's vestibular tests (due to a transient labyrinthine hypofunction), the presence of co-existing diseases, the underestimation of the incidence of acute attacks not reported by the patient (because were attributed to other causes) and finally wrong diagnostic approaches. There- 
fore it is necessary a correct initial symptoms assessment for an early identification of MD. Currently the majority of audiological tests have negative or doubt results for a definite diagnosis in the initial stage, however early diagnosis make possible to treat the disease before becomes chronic. Peritympanic electrocochleography (P.EcochG) is a non invasive method that can identify hydrops, an indispensable condition although not sufficient by itself for a definite diagnosis in all MD's patients.

P.EcochG testing has advantages, such as not being invasive and painless, but also has disadvantages. The major disadvantage is that the far distance of the recording electrode to the response generators provides for a very unfavorable signalto-noise ratio so recordings require more signal averaging and the components tend to be lower in amplitude. However it is. The response parameters of P.Eochg are: 1) cochlear microphonics, 2) summating potentials (SP), and 3) action potentials (AP). The cochlear microphonics and the SP represent the bioelectric activity of the cochlea, instead the AP refers to the activity of distal afferent fibers of the 8th nerve. In our study the only parameter considered was the SP/AP ratio, due to the simplicity and directness of its analysis according also to the literature $[6,7]$. An increased level of SP/AP amplitude ratio points to the diagnosis of MD (Fig. 1). SP/AP ratio higher than 0.37 were considered positive for the presence of endolymphatic hydrops [8,9]. The sensivity described is up to $60 \%$ [10]. SP amplitude was not used because there were considerable overlap results between patients with $\mathrm{MD}$, normal hearing or cochlear hearing loss (sensitivity no higher than $30 \%$ ) [11]. Further measures have been suggested to improve the sensitivity area ratio [12], latency difference stimuli of opposite polarity [13], Meniere's Disease Index or MDI [5] however the main drawback is that these procedures are not easily performed and is unclear whether the inclusion of this measure provides a substantial advantage [14]. The aim of this study is to evaluate the clinical usefulness of the P.EcochG as an early objective diagnostic tool for MD, always in accordance with the criteria of the American Academy of Otolaryngology-Head and Neck Surgery (AAO-HNS) 1995. Moreover to study the utility of the examination, even as a follow up option, in the patients with a single complete presentation (which classifies them as probable MD and could evolve in defined MD), taking in consideration as the only parameter, the abnormal results of the P.EcochG SP/AP ratio. In our study the cut-off limit pointing to a defined MD, was an $\mathrm{SP} / \mathrm{AP}$ ratio higher than 0.37 .
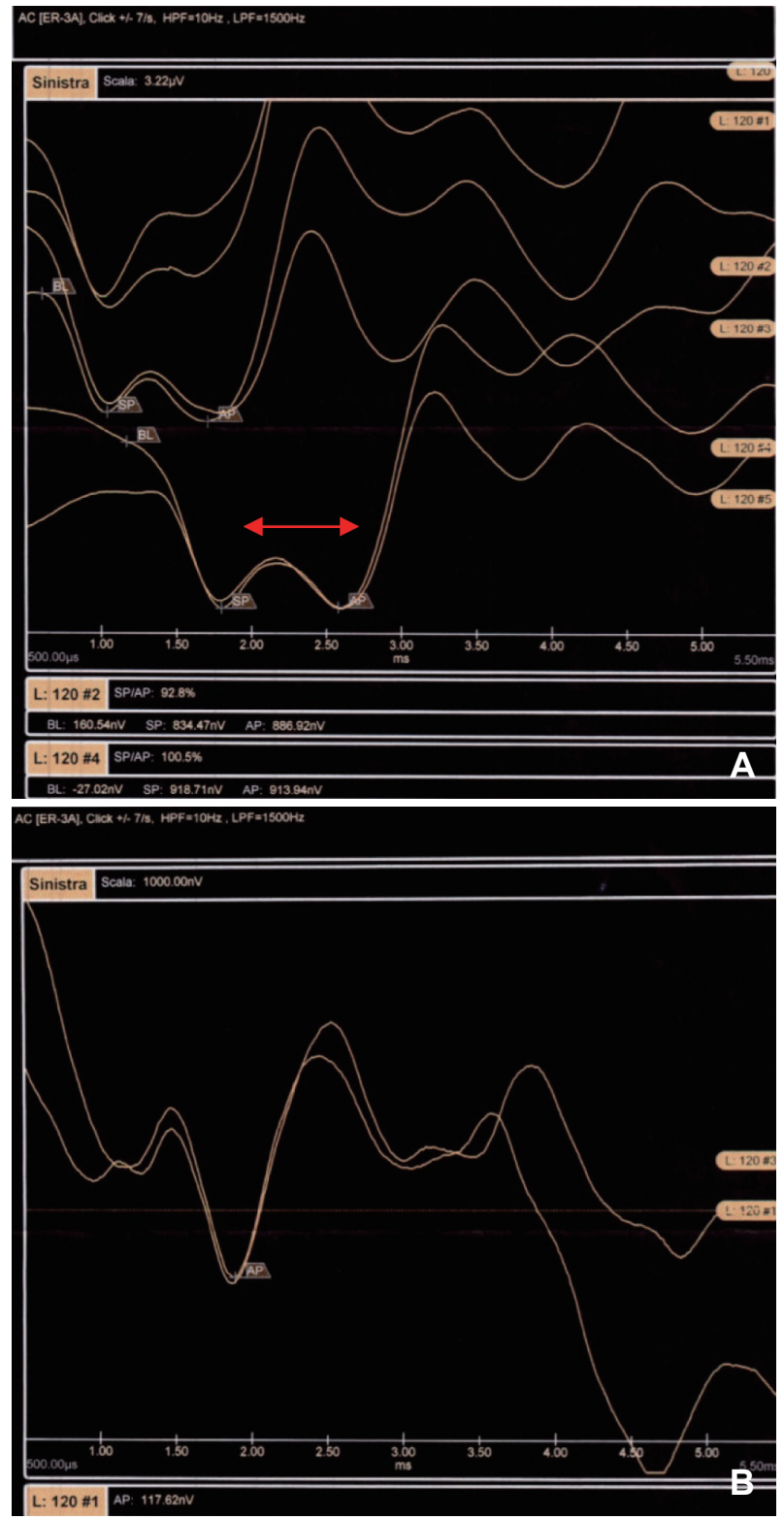

Fig. 1. Example of SP/AP tracings for the two groups. A: Shows an increased level of SPIAP (>0.37) amplitude ratio in defined MD patients. B: Shows a normal amplitude ratio in control group, classified as probable MD patients. Note the clear difference between the SP/AP tracings in the upper trace (red arrows). SP: summating potentials, AP: action potentials, MD: Meniere's disease.

\section{Subjects and Methods}

The study population was composed of 34 subjects referred to the audiology department of the San Camillo Forlanini Hospital in 3-year period, between 2011-2013. The inclusion criteria are the presence of complete symptoms compatible with MD (tinnitus, hearing loss and vertigo) associated by documented unilateral sensorineural hearing loss. The exclusion criteria are: incomplete presentation; complete presen- 
tation associated to conductive, mixed or retrocochlear hearing loss; complete presentation, characterized by vertigo syndromes that are not compatible for clinical features and duration for MD. Other clinical exclusion criteria are tympanic perforations, active infections, inflammatory and non inflammatory stenosis (exostoses, osteomas) that make impossible to perform the P.EcochG. The first patient group (study group) was composed of 7 male and 6 female and all had recurrent complete symptoms. The mean age was 51.7 years (22 -77), average age lower in males (48.7 years/22-77) compared to females (54.7 years/39-75). All patients were submitted to medical history collection, immunologic tests and complete functional otologic examination [pure-tone audiometry, speech audiometry, impedance, caloric test, Head Impulse Test, auditory brainstem response, cervical-vestibular evoked

Table 1. Various types of immunology, audiology and imaging tests conducted in both study groups

\begin{tabular}{lc}
\hline \multicolumn{1}{c}{ Test categories } & \multicolumn{1}{c}{ Tests } \\
\hline Immunology tests & $\begin{array}{c}\text { ANA, ASMA, ANCA, anti cardiolipin, } \\
\text { anti phospholipids, LAC } \\
\text { Specific IgG and IgM } \\
\text { EBV, Toxoplasma, mumps, varicella, } \\
\text { herpes 1, enteroviruses } \\
\text { Audiology tests }\end{array}$ \\
& $\begin{array}{l}\text { Otoscopy, pure-tone audiometry, } \\
\text { speech audiometry, impedance, } \\
\text { caloric test, HIT, ABR, C-VEMPS, } \\
\text { P.EcochG }\end{array}$ \\
Imaging & MRI
\end{tabular}

ANA: antinuclear, ASMA: anti-smooth muscle, ANCA: cytoplasmatic anti-neutrophil, LAC: test for lupus anticoagulant, HIT: Head Impulse Test, ABR: auditory brainstem response, CVEMPS: cervical-vestibular evoked myogenic potentials, MRI: magnetic resonance imaging, P.EcochG: peritympanic electrocochleography, EBV: Ebstein Barr virus myogenic potentials (P.EcochG)] and MRI (Table 1). A Labat system (Epic Plus 16-bit; LABAT INTERNATIONAL SPL, Mogliano Veneto, Italy) was used to record P.EcochG potentials. P.EochG was recorded within the first 24 hours from first complete clinical presentation, even if at this stage suggests a probable MD and certainly not a definite early stage of the disease. The only parameter that was taken in consideration during the study, was the SP/AP ratio. The acoustic stimulus was used alternately with the click rate of $7.1 \mathrm{sec} .110 \mathrm{~dB}$ sound pressure level in monaural presentation via earphones. The filters were set in the range 10-2,000 Hz. An average of 2,000 tracks per exam were recorded. For recording we use a ballon electrode surrounded by cotton (it's possible to homemade or available to buy). The correct position can be confirmed objectively by monitoring the noise floor of the EEG. Patients groups were subdivided into three categories for each group based on the type of audiometric curve (A low-tone frequency/B flat/C high-tone) (Table 2), in order to observe any correlation between the various audiometric curves and the SP/AP ratio regarding the progression of the disease and as it was mentioned in the introduction [2-4]. Every patients had a low-moderate degree of hearing loss (no higher than 70 $\mathrm{dB}$ HL). A second group (control group) of 21 patients (complete symptoms compatible with MD but with only one presentation which classified them as a probable MD) was included in the study.

\section{Results}

The progression in defined MD is demonstrated in Table 3. Seven subjects out of 13 satisfy the diagnostic MD clinical criteria which classified them as defined MD. The analysis

Table 2. The two patient groups classified into three audiometric categories for each group: low tone frequency (A), flat (B), and hightone $(\mathrm{C})$

\begin{tabular}{lcc}
\hline \multicolumn{1}{c}{ Group } & Patients & Audiogram shape-patients \\
\hline Recurrent complete symptoms & 13 & A-3 \\
& & B-8 \\
Episodic complete symptoms (probable MD, control group) & 21 & C-2 \\
& & B-10 -5 \\
\hline
\end{tabular}

MD: Meniere's disease

Table 3. Results considering the parameter SP/AP ratio (>0.37), for six out of seven clinically defined MD patients: low tone frequency $(\mathrm{A})$, flat (B), and hightone (C)

\begin{tabular}{cccc}
\hline Group & Clinicaly defined MD & SP/AP $>0.37$ & Audiogram shape-patients \\
\hline Recurrent complete symptoms & 3 & 3 & A-3 \\
& 4 & 3 & B-8 \\
& 0 & 0 & C-2 \\
\hline
\end{tabular}

SP: summating potentials, AP: action potentials, MD: Meniere's disease 
of the SP/AP results shows high predictability for the MD (SP/ $\mathrm{AP}>0.37$ in six out of seven cases with a sensitivity greater than $80 \%$ ), providing a further confirmation for an early defined MD diagnosis. Only one clinical defined MD case had a normal SP/AP ratio (0.21). Regarding the other 6 out of 13 subjects, a different diagnosis than MD was made which explained better their symptoms. Among twenty-one subjects in the control group, only one patient had positive P.EcochG results. Even if he couldn't be classified as defined MD (which was impossible as the presence of at least two episodes is essential), he probably had an atypical or incomplete MD. This hypothesis was made because collected data of this patient for the SP/AP ratio was frankly positive (0.90), much higher than our cut-off limit (0.37).

\section{Discussion}

The complexity of the diagnostic process in MD is indisputable as documented by the succession of different MD classifications proposed. The most common classification (AAOHNS, 1995), despite its widespread nature, does not appear completely satisfactory as shown a revision proposed in the 2015 [15]. To confirm this, a retrospective analysis of cases proposed by Ménière [16] itself raised doubts about their correct classification with a strong clinical suspicion of intralabyrinthine hemorrhage in a patient [17]. It seems clear that in such a heterogeneous degree of uncertainty it is necessary to support clinical suspect with additional exams. Although none of the subjects in the control group satisfy the necessary criteria, one patient had an abnormal P.EcochG (frankly positive for hydrops) compatible with signs of MD, confirming the occasional pittfalls of the current clinical criteria. Only one MD patient had normal P.EcochG. The purpose of our work is to analize the benefits of P.E cochG testing in early MD diagnosis, eventhough in these cases can be useful test repeating as a follow up. P.EcochG identifies hydrops in more than $80 \%$ MD's patients. The analysis of the test predictability showed high compatibility with MD defined diagnosis and only one false negative result (SP/AP ratio 0.21) that maybe could be explained with a cleft in the Reissner's membrane, resulting to a loss of endolymph [18]. More than 50\% of the patients with recurrent complete Meniere-like presentation receive a defined MD diagnosis, however it isn't uncommon that another diagnosis could give a better explanation for their symptomatology. Six patients of the study group had these diagnosis: 3 patients had autoimmune disorders which exlude MD diagnosis, 1 patient was diagnosticated with basilar migraine and 2 patients suffered a recurrent sudden hearing loss. Besides the already discussed limitations of the absence of a shared procedure (electrode placement or method) the major debated point is the SP/AP ratio cut-off (between $0.32-0.5$ in literature, reasonably no higher than 0.4 ). Finally it is important to remember that the presence of hydrops is considered "necessary but not sufficient" for the diagnosis of MD and cannot be used to diagnosis alone.

In conclusion, it is currently difficult to identify the early manifestations of MD, even with a complete presentation of the classic "triad." Not always recurrent presentations of one or more symptoms of the triad are due to MD and a differential diagnosis is always nessessary. P.EcochG is a non invasive test that can identify hydrops, a necessary condition that can help in early diagnosis. SP/AP ratio is the easiest parameter to define. Our study demonstrates a satisfactory sensibility percentage in recurrent MD presentation ( $>80 \%$ ). Early identification of MD can lead to an early treatment of the disease in a reversible fase.

\section{Conflicts of interest}

The authors have no financial conflicts of interest.

\section{REFERENCES}

1) Belinchon A, Perez-Garrigues H, Tenias JM. Evolution of symptoms in Ménière's disease. Audiol Neurootol 2012;17:126-32.

2) Junicho M, Aso S, Fujisaka M, Watanabe Y. Prognosis of low-tone sudden deafness-does it inevitably progress to Meniere's disease? Acta Otolaryngol 2008;128:304-8.

3) Thomas K, Harrison MS. Long-term follow up of 610 cases of Ménière's disease. Proc R Soc Med 1971;64:853-7.

4) Enander A, Stahle J. Hearing in Menière's disease. A study of puretone audiograms in 334 patients. Acta Otolaryngol 1967;64:543-56.

5) Claes GM, De Valck CF, Van de Heyning P, Wuyts FL. The Ménière's Disease Index: an objective correlate of Ménière's disease, based on audiometric and electrocochleographic data. Otol Neurotol 2011;32: 887-92.

6) Kimura H, Aso S, Watanabe Y. Prediction of progression from atypical to definite Ménière's disease using electrocochleography and glycerol and furosemide tests. Acta Otolaryngol 2003;123:388-95.

7) Chung WH, Cho DY, Choi JY, Hong SH. Clinical usefulness of extratympanic electrocochleography in the diagnosis of Ménière's disease. Otol Neurotol 2004;25:144-9.

8) Orchik DJ, Shea JJ Jr, Ge X. Transtympanic electrocochleography in Menière's disease using clicks and tone-bursts. Am J Otol 1993;14: 290-4.

9) Pou AM, Hirsch BE, Durrant JD, Gold SR, Kamerer DB. The efficacy of tympanic electrocochleography in the diagnosis of endolymphatic hydrops. Am J Otol 1996;17:607-11.

10) Coats AC. The summating potential and Meniere's disease. I. Summating potential amplitude in Meniere and non-Meniere ears. Arch Otolaryngol 1981;107:199-208.

11) Mori N, Asai H, Doi K, Matsunaga T. Diagnostic value of extratympanic electrocochleography in Menière's disease. Audiology 1987; 26:103-10.

12) Ferraro JA, Tibbils RP. SP/AP area ratio in the diagnosis of Ménière's disease. Am J Audiol 1999;8:21-8.

13) Orchik DJ, Ge NN, Shea JJ Jr. Action potential latency shift by rarefaction and condensation clicks in Menière's disease. J Am Acad Audiol 1998;9:121-6. 
14) Santarelli R, Arslan E. Electrocochleography in auditory neuropathy. Hear Res 2002;170:32-47.

15) Lopez-Escamez JA, Carey J, Chung WH, Goebel JA, Magnusson M, Mandalà $\mathrm{M}$, et al. [Diagnostic criteria for Menière's disease. Consensus document of the Bárány Society, the Japan Society for Equilibrium Research, the European Academy of Otology and Neurotology (EAONO), the American Academy of Otolaryngology-Head and Neck Surgery (AAO-HNS) and the Korean Balance Society]. Acta Otorrinolaringol Esp 2016;67:1-7.
16) Ménière P. Mémoire sur des lésions de l'oreille interne donnant lieu à des symptômes de congestion cérébrale apoplectiforme. Gaz Méd Paris 1861;3:597-601.

17) Conlon BJ, Gibson WP. Electrocochleography in the diagnosis of Meniere's disease. Acta Otolaryngol 2000;120:480-3.

18) Zenner HP, Reuter G, Zimmermann U, Gitter AH, Fermin C, LePage EL. Transitory endolymph leakage induced hearing loss and tinnitus: depolarization, biphasic shortening and loss of electromotility of outer hair cells. Eur Arch Otorhinolaryngol 1994;251:143-53. 